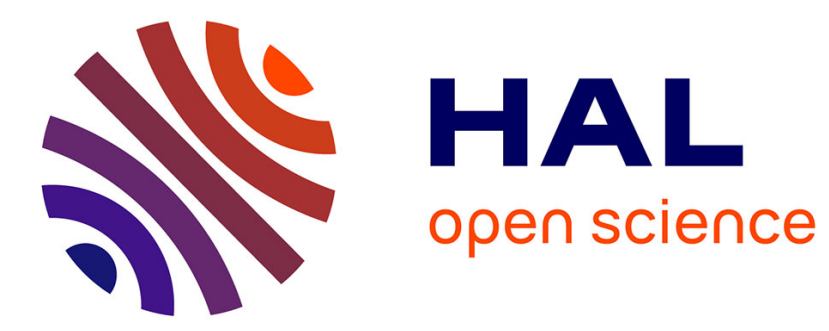

\title{
Ressources en eau et glissements de terrain: exemple du bassin versant de la Semoigne (bassin de Paris, France)
}

Nicolas Bollot, Alain Devos, Guillaume Pierre

\section{To cite this version:}

Nicolas Bollot, Alain Devos, Guillaume Pierre. Ressources en eau et glissements de terrain: exemple du bassin versant de la Semoigne (bassin de Paris, France). Géomorphologie: relief, processus, environnement, 2015, Actes des 15e Journées des Jeunes Géomorphologues, 21 (1), pp.7-20. 10.4000/geomorphologie.10826 . hal-03277178

\section{HAL Id: hal-03277178 \\ https://hal.science/hal-03277178}

Submitted on 2 Jul 2021

HAL is a multi-disciplinary open access archive for the deposit and dissemination of scientific research documents, whether they are published or not. The documents may come from teaching and research institutions in France or abroad, or from public or private research centers.
L'archive ouverte pluridisciplinaire HAL, est destinée au dépôt et à la diffusion de documents scientifiques de niveau recherche, publiés ou non, émanant des établissements d'enseignement et de recherche français ou étrangers, des laboratoires publics ou privés. 


\title{
Ressources en eau et glissements de terrain : exemple du bassin versant de la Semoigne (bassin de Paris, France) Water resources and landslides: the case study of the Semoigne catchment (Paris basin, France)
}

\author{
Nicolas Bollot*, Alain Devos*, Guillaume Pierre*
}

\begin{abstract}
Résumé
Dans le bassin versant de la Semoigne, les glissements de terrain anciens connaissent des réactivations superficielles, dont le fonctionnement, notamment en ce qui concerne la circulation de l'eau en leur sein, est peu connu. Une approche hydrologique, basée sur l'identification des niveaux aquifères, la cartographie des sources et la spatialisation des ressources en eau à partir de campagnes de mesures sérielles, croisée à une cartographie géomorphologique, permet de préciser les interactions entre aquifères et glissements de terrain. Les résultats montrent que la vidange aquifère s'effectue essentiellement dans la vallée de la Semoigne qui concentre, dans sa partie aval, les volumineux glissements de terrain hérités d'une période cataglaciaire à surplus hydrologique marqué. Les réactivations holocènes sont également corrélées à la vidange aquifère plutôt qu'à l'infiltration directe des précipitations.
\end{abstract}

Mots clés : ressources en eau, glissements de terrain, bassin versant, chronologie, Semoigne.

\begin{abstract}
The old landslides of the Semoigne catchment exhibit superficial reactivations, which mechanism is not well-known, in particular the water pathway within the internal body of the landslide. Hydrological approach is based on the identification of the aquifer levels, the cartography of the springs and the spatial characteristics of the water resources derived from serial hydrological measures. Cross-correlation of hydrological and geomorphological data points out the interactions between aquifer and mass movement. The draining of the aquifer essentially occurs in the lower Semoigne valley, where large inherited landslides remain. If cataglacial period account for hydrological excess leading to slope failure, Holocene reactivations are also linked with groundwater supply rather than with infiltration of rain water.
\end{abstract}

Key words: water resources, landslides, Semoigne catchment, chronology.

\section{Abridged English Version}

Among recent works, landslide activity is often examined using electrical resistivity tomography (Grandjean et al., 2006), hydric state of the material being correlated with rainfall (Grandjean et al., 2009; Lebourg et al., 2010; Ruiz-Villanova et al., 2011; Belle et al., 2013). The relationship between rainfall and piezometric surface data within the internal body of the landslide (Lissak et al., 2009), or in unstable bedrocks (Pierre and Lahousse, 2006) has also been studied in detail. On the contrary, very few studies focused on hydrogeological exchange between bedrock and superficial deposits (Compagnon et al., 1997; Guglielmi et al., 2005; Lee et al., 2008). Meanwhile, a recent paper focuses on the importance of landslide groundwater and its interactions with the subsurface (Parriaux, 2010). The following hydrological approach allows to highlight the role of groundwater on the occurrence and the distribution of the landslides, and also to reconsider susceptibility assessments, adding water resources to structural and cyclic parameters usually used for predicting models (Van den Eeckhaut et al., 2010; Conforti et al., 2014; Umar et al., 2014).

The small Semoigne catchment $\left(100 \mathrm{~km}^{2}\right)$ is situated on the back slope of the Ile-de-France cuesta (fig. 1). Unconformably overlying the Campanian chalk, Tertiary strata have a $0.2 \% \mathrm{~W}$ dip, and the succession of facies from bottom to top determine a main aquifer (Ypresian sands and middle and lower Lutetian coarse limestone) and several perched aquifers in the permeable levels of upper Lutetian (Marnes et caillasses), Auversian (cornestone limestone) and Ludian (marl and limestone) (fig. 2). The network of hydrological measures (conductivity, temperature and discharge) takes into account the 14 elementary basins of the Semoigne catchment (fig. 3). The measurement campaigns

\footnotetext{
* Université de Reims Champagne-Ardenne - GEGENA² EA 3795 - Département de Géographie - 57 rue Pierre Taittinger - 51096 Reims Cedex France (nicolas.bollot@univ-reims.fr, alain.devos@univ-reims.fr, guillaume.pierre@univ-reims.fr).
} 
were achieved during summer and winter low water periods (fig. 4 and fig. 5). The results (identification of aquifers and aquicludes, typology of springs, spatialization of water resources) are cross-correlated with geomorphological data to highlight the potential interactions between aquifer and landslides (fig. 6 and fig. 7). Hydrological profiles enhance low water resource and highly mineralized water in the upper catchment, and increasing discharge as well as diminishing conductivity in the lower catchment. The draining of the aquifer essentially occurs in the lower Semoigne valley, through the stream bed or the valley-side springs which continuously supply water, possibly leading to slope instability. The downhill movement of surface material creates some discontinuities in the permeability. As a result, on the right valley side of the lower Semoigne, the draping of the intersection of the water-table and ground surface explains anomalies in springs position, while on the opposite valley side, corresponding to the inner bank, groundwater flows along the potential spring-line (fig. 8).

The state of groundwater resources depends on the hydrological balance, infiltration being ensured during phases of hydrological excess. Such conditions, linked to moderate actual evapotranspiration and high effective rainfall, are exacerbated by the thaw of the permafrost during cataglacial periods, whereas the hydric (and hydrologic) deficit increases during the subsequent temperate climates. Therefore, the destabilization of the slopes must have been synchronous with the degradation of the permafrost and with the restoration of the aquifer. This took place during the transition period between Lateglacial and Holocene (Pánek et al., 2010). The "old" mass movements of the Champagne vineyard must then be of Pleistocene age, and not necessarily of Preglacial age (Van den Eeckhaut et al., 2010), for along the Semoigne river, the landslides reach the valley floor which is connected with the postglacial alluvial plain of the Marne river. These facts incite to add groundwater as a parameter in landslide susceptibility assessments (Schmidt and Dikau, 2004), as it is at least as important as lithology and slope gradient (Bălteanu et al., 2010; Van den Eeckhaut et al., 2010; Conforti et al., 2014). Moreover, slope gradient is not a pertinent parameter when susceptibility maps concern area already affected by landslides, where forces promoting instability have been reduced. Consequently, only processes of reactivation must be assessed.

Superficial reactivation of old landslides of the Champagne vineyard has been assigned to rainfall and infiltration (Guéremy and Vejux, 1987; Marre, 1987). However, the comparison between the piezometric level, the cumulative sum of precipitation and the occurrence of mass movements show that heavy rainfalls do not induce slope failures if the groundwater level is low, and also that the water content of the landslide is mainly controlled by groundwater discharge (fig. 9). Nevertheless, Holocene climatic fluctuations have promoted slope instability, especially at the upper Atlantic-Subboreal boundary (González-Díez et al., 1999; Soldati et al., 2004), period from which anthropogenic influence contributes to increased slope instability (Dapples et al., 2002; Remondo et al., 2005). The Semoigne case study shows that the hydrolo- gic balance must be substituted for hydric balance to explain reactivations: in the Champagne vineyard, where human actions are related to intensification of viticulture rather than to improvement of slope stability, the water content of the slipped material and the reactivation of landslides are mainly controlled by groundwater supply, so that heavy rainfall is not necessarily a triggering factor.

\section{Introduction}

Les glissements de terrain ont fait l'objet d'un grand nombre de publications, et d'une multiplicité d'approches dont le point commun est la compréhension des mécanismes engendrant le mouvement. Parmi les travaux les plus récents, l'état hydrique des matériaux appréhendé par imagerie électrique (Grandjean et al., 2006) est le plus souvent corrélé aux précipitations (Grandjean et al., 2009 ; Lebourg et al., 2010 ; Ruiz-Villanova et al., 2011 ; Belle et al., 2013). Les relations entre pluviométrie et fluctuations du niveau de la nappe comprise dans les mouvements de terrain (Lissak et al., 2009) ou dans des substrats instables (Pierre et Lahousse, 2006) ont également fait l'objet d'études détaillées. Les relations hydrogéologiques entre substrat et formations superficielles sont moins souvent abordées (Compagnon et al., 1997 ; Guglielmi et al., 2005 ; Lee et al., 2008), bien que l'importance du comportement hydrodynamique des aquifères sur la stabilité des versants déjà affectés par des mouvements de terrain ait fait l'objet d'une mise au point récente (Parriaux, 2010). L'approche hydrologique qui suit conduit à s'interroger sur le rôle des eaux souterraines dans l'occurrence et la répartition des glissements de terrain, et à reconsidérer la question de la susceptibilité au glissement, en ajoutant aux paramètres structurels et conjoncturels habituellement pris en compte dans les modélisations (Van den Eeckhaut et al., 2010 ; Conforti et al., 2014 ; Umar et al., 2014) les ressources en eaux et leurs relations avec la répartition spatiale des glissements de terrain.

\section{Contexte morphostructural et méthodologie}

En rive droite de la vallée de la Marne $(65 \mathrm{~m})$ et à une vingtaine de kilomètres en aval d'Epernay, le bassin versant de la Semoigne draine, sur une surface de $100 \mathrm{~km}^{2}$, le revers de la cuesta d'île-de-France (plateau briard, $220 \mathrm{~m}$; fig. 1). La couverture tertiaire, discordante sur la craie campanienne, est constituée d'une succession de faciès meubles et cohérents, déterminant en outre un aquifère principal (sables de l'Yprésien et Calcaires grossiers du Lutétien inférieur et moyen) et plusieurs aquifères perchés dans les niveaux semiperméables du Lutétien supérieur (Marnes et caillasses), de l'Auversien (calcaires gréseux) et du Ludien (marnes et calcaires). L'ensemble est recouvert par des argiles à meulière issues d'une météorisation chimique polyphasée pré-stampienne puis quaternaire jalonnant une surface infra-stampienne exhumée («surface de Brie »; Dewolf et Pomerol, 1997). Le pendage ouest des assises tertiaires est de l'ordre de $0,2 \%$, et une ondulation anticlinale parallèle à la vallée de la Marne 


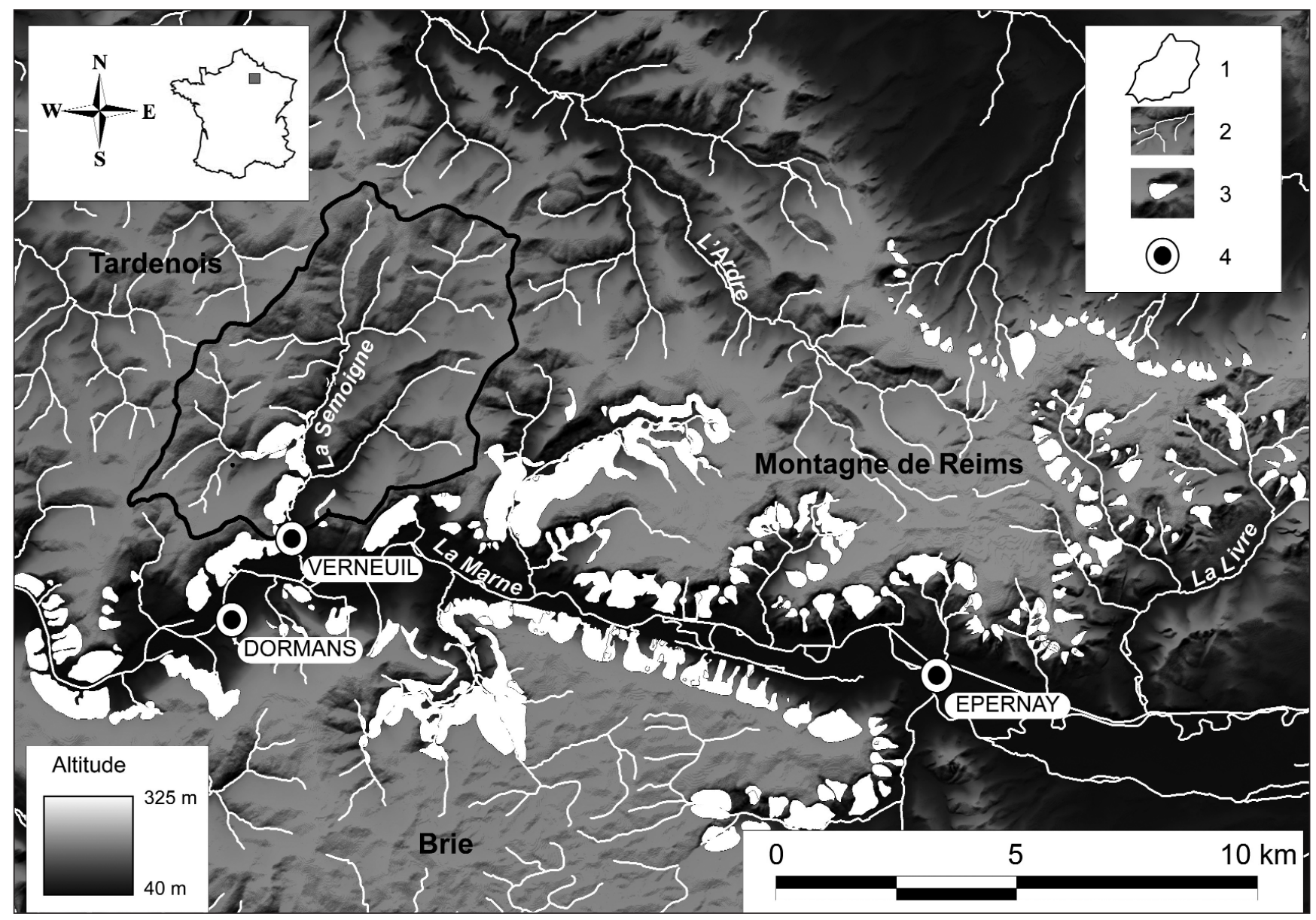

Fig. 1 - Carte de localisation. 1 : bassin versant de la Semoigne ; 2 : cours d'eau ; 3 : glissement de terrain ; 4 : localité.

Fig. 1 - Location map. 1: Semoigne catchment area; 2: stream; 3: landslide; 4: locality.

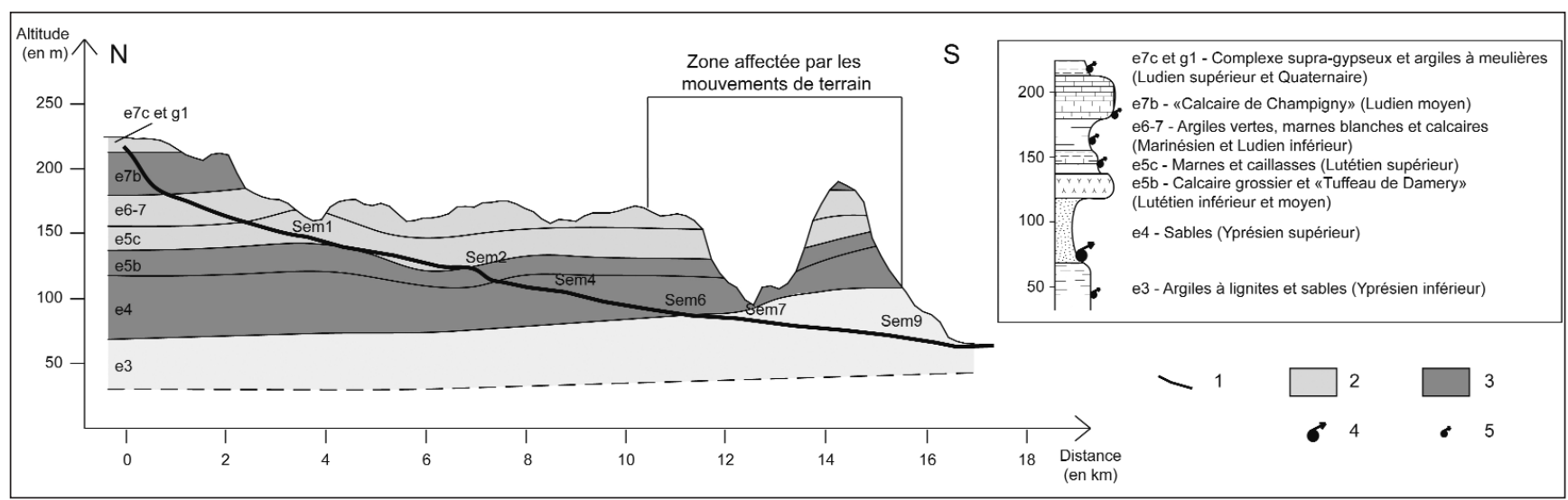

Fig. 2 - Coupe géologique et profil en long de la Semoigne. 1 : profil en long ; 2 : formations semi-perméables ; 3 : principaux aquifères ; 4 : source de dépression de l'aquifère principal ; 5 : source de trop-plein perché.

Fig. 2 - Geological section and Semoigne longitudinal profile. 1: river profile; 2: semi-permeable layers; 3: main aquifers; 4: depression spring of main aquifer; 5: perched overflow spring.

détermine un contre-pendage des couches à la confluence de la Semoigne et de la Marne (fig. 2). À cette structure s'ajoute une fracturation de détente mécanique liée à l'incision des vallées (Devos et al., 2012 ; Bollot et al., 2014).

Le bassin versant de la Semoigne est utilisé comme bassin atelier car il est homogène, de taille modeste $\left(100 \mathrm{~km}^{2}\right)$, et il concentre dans sa partie aval une dizaine de glissements de terrain. La cartographie des ressources en eau au sein d'un bassin versant repose sur l'organisation spatiale des écoulements des cours d'eau en phase d'étiage. En effet, ces écoulements sont représentatifs des conditions de vidange aquifère et de l'état des réserves en eau. Compte tenu de leur petite 
taille $\left(<300 \mathrm{~km}^{2}\right)$, les écoulements des bassins homogènes tels que celui de la Semoigne ne sont pas systématiquement surveillés, ou au mieux à partir d'une seule station hydrométrique. Une série de mesures a donc été effectuée à partir d'un réseau constitué de 6 points sur le drain principal et de 8 points sur les principaux affluents, ce qui correspond à une densité moyenne de 1 point pour $7 \mathrm{~km}^{2}$ de surface (fig. 3 ). Deux campagnes de mesures sérielles de débits et de paramètres physico-chimiques ont été réalisées en situations hydrologiques stables de basses-eaux (absences prolongées de précipitations, phase de tarissement), les unes en saison froide (mars 2014) et les autres en saison chaude (septembre 2013), de manière à spatialiser la vidange aquifère pour des situations hydrologiques contrastées mais uniformes. Chaque campagne a été réalisée en moins de 12 heures afin de s'inscrire dans une période non influencée par une vitesse de tarissement trop rapide. Deux autres campagnes de mesures physico-chimiques ont été réalisées en saison chaude, le 26/09/2013 et durant une journée de gel, le 12/12/2013.

Les paramètres physico-chimiques mesurés sont la conductivité électrique à $25^{\circ} \mathrm{C}$ et la température. Ces deux paramètres permettent de distinguer les eaux de nappe des eaux de ruissellement : la conductivité témoigne de la minéralité totale des eaux qui dépend de la solubilité des roches encaissantes et du temps de résidence des eaux dans la zone saturée, tandis que la température permet d'opposer les eaux de nappes homothermes des eaux de ruissellement qui enregistrent les amplitudes thermiques atmosphériques nycthémérales. Les mesures sont faites avec une perche de jaugeage à intégration équipée d'un micro-moulinet de type C2 pour les débits, et avec une sonde multiparamètre WTW pour les conductivités et les températures. Les résultats sont valorisés par des profils hydrologiques en débits bruts, en débits spécifiques, en conductivités électriques et en températures et sont cartographiés par bassins élémentaires. Parallèlement à ces campagnes de mesures, une cartographie des sources a été réalisée sous SIG à partir des cartes géologiques à 1 : 50000 de la Banque du Sous-Sol (BSS) et d'inventaires du BRGM, complétés par une vérification sur le terrain. Cette cartographie a pour objectif une comparaison (par superposition de couches sous SIG) de l'organisation spatiale des émergences de nappe et des mouvements de terrain. Ces derniers ont systématiquement été cartographiés dans la partie aval du bassin versant de la Semoigne, où ils abondent, à partir de données de terrain : indices topographiques, affleurements, occupation des sols (cultures et zones humides).

\section{Résultats}

Les profils hydrologiques illustrent l'évolution spatiale des débits bruts et spécifiques de la Semoigne de l'amont vers l'aval, en fonction de la surface cumulée des sous-bassins versants. Les deux profils de débits bruts (fig. 4A) présentent une évolution linéaire corrélative de l'augmenta- tion de la surface drainée, ce qui témoigne a priori d'une vidange aquifère relativement homogène dans le bassin versant (contrairement à ce que l'on observe le long des cours d'eau drainant les calcaires jurassiques du bassin de Paris ; Devos et al., 1999, 2007, 2013), et ce qui montre en outre la permanence du comportement hydrodynamique du bassin pour des situations, stabilisées mais contrastées, de basses eaux. En revanche, les profils hydrologiques réalisés à partir des valeurs de débits spécifiques sont plus contrastés. En effet, pour la campagne réalisée en étiage plus sévère (septembre 2013), deux parties du bassin s'opposent : en amont du point Sem 4, les débits spécifiques sont particulièrement indigents (moins de $1 \mathrm{l} / \mathrm{s} / \mathrm{km}^{2}$ ) alors qu'ils se stabilisent autour de 2,5 1/s/km² entre les points Sem 4 et Sem 9.

Les quatre profils de conductivité (fig. 4C) présentent des valeurs élevées (> 700 $\mathrm{S} / \mathrm{cm}$ ) comparativement à celles mesurées dans les calcaires jurassiques ou la craie du Crétacé (Lejeune et Devos, 2004 ; Devos et al., 2013), ce qui témoigne d'eaux fortement minéralisées, voire saturées. Ces valeurs sont attribuables aux ions carbonatés des nappes contenues dans les formations du Ludien et du Lutétien supérieur (fig. 2). Les eaux des Marnes et caillasses du Lutétien supérieur enregistrent d'ailleurs les valeurs les plus fortes (800 à $900 \mu \mathrm{S} / \mathrm{cm})$. Si les valeurs restent fortes tout le long du drain principal, l'évolution de la conductivité, quasi similaire pour les 4 campagnes de mesures, montre des contrastes modérés mais significatifs de minéralité entre l'amont et l'aval. Dans la partie supérieure du bassin, les valeurs augmentent légèrement de 30 à $40 \mu \mathrm{S} / \mathrm{cm}$ entre Sem 1 et Sem 4 avant de diminuer d'autant en aval (fig. 5B- 


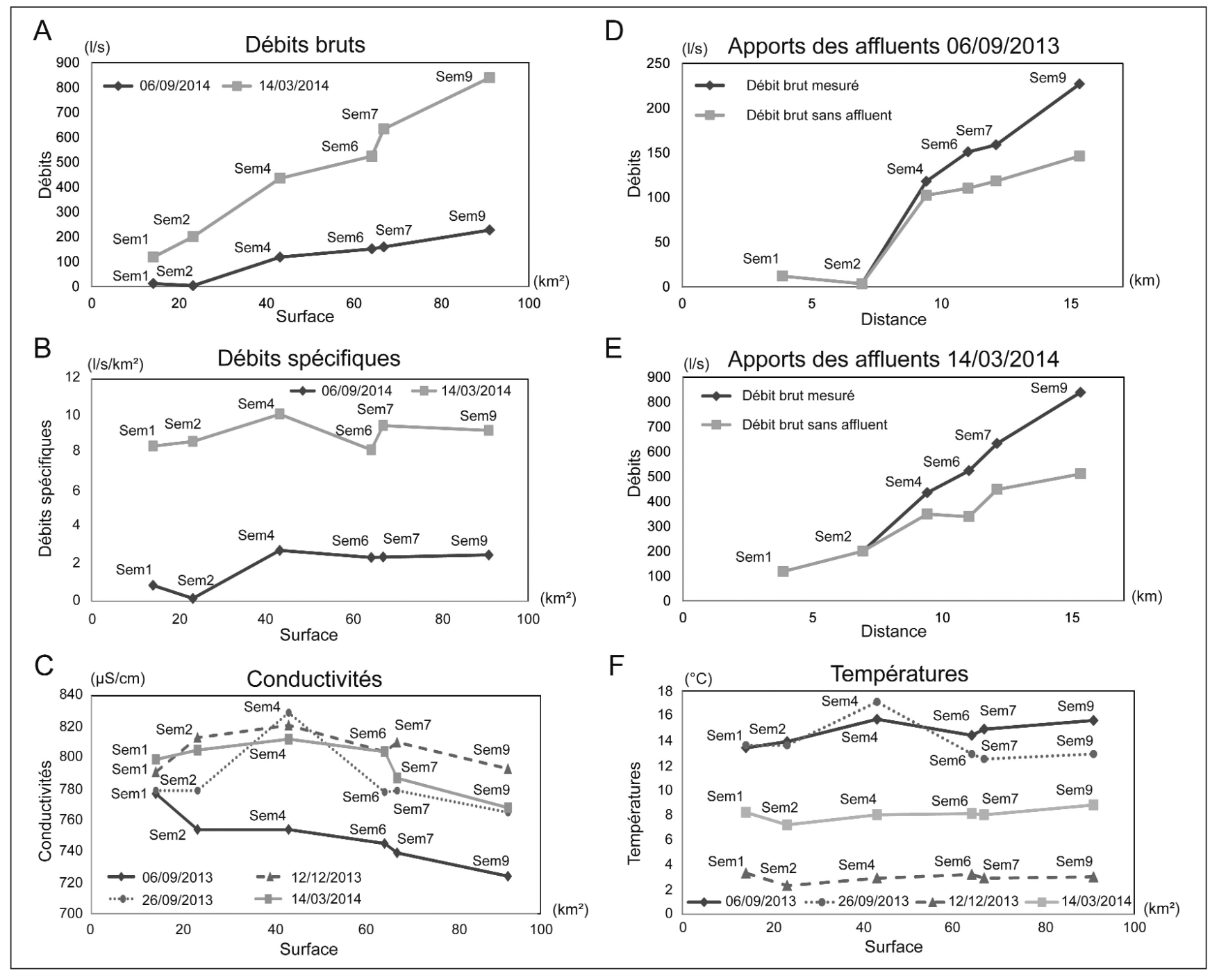

Fig. 4 - Profils hydrologiques de la Semoigne.

Fig. 4 - Hydrologic profiles of the Semoigne river.

E). Seule la campagne réalisée en étiage sévère (06/09/2013) n'obéit pas à cette règle, ce qui s'explique aisément par l'indigence des écoulements en amont (Sem 1 et Sem 2) induisant une grande vulnérabilité à la pollution, susceptible d'influencer les résultats.

Les profils thermiques des 4 campagnes de mesures sont plus contrastés dans le temps que dans l'espace (fig. $4 \mathrm{~F}$ et fig. 5G-J). En effet, les amplitudes maximales entre les campagnes sont de $10^{\circ}$ à $14^{\circ} \mathrm{C}$ aux mêmes points, ce qui souligne a priori une forte dépendance des conditions atmosphériques et un faible apport de nappe. En revanche, dans l'espace, pour une même campagne, les contrastes thermiques restent faibles $\left(<5^{\circ} \mathrm{C}\right.$; fig. $\left.5 \mathrm{G}-\mathrm{J}\right)$. Dans les cours d'eau drainant les nappes des calcaires jurassiques, on observe classiquement une homothermie au niveau des recoupements aquifères et une forte vulnérabilité aux conditions atmosphériques pour les cours d'eau filtrants, ce qui détermine de forts contrastes spatiaux des valeurs mesurées. Dans le cas du bassin de la Semoigne, les étangs piscicoles du domaine de Neuville situés dans une zone d'apport hydrolo-

gique en amont immédiat du point Sem 4 (où les débits spécifiques augmentent sensiblement) tendent à lisser les profils thermiques des eaux. Lors la campagne du 12/12/2013, réalisée un jour de gel, la température de la Semoigne n'augmente que de $0,9^{\circ} \mathrm{C}$ dans la traversée des étangs alors qu'elle augmente de $3,5^{\circ} \mathrm{C}$ durant la campagne du 26/09/2013 où la température atmosphérique a atteint $25^{\circ} \mathrm{C}$.

L'analyse des profils de débit, de conductivité et de température montre que les écoulements de la Semoigne sont marqués dans la partie amont par de faibles ressources en eau mais une forte minéralité, alors que dans la partie aval les débits augmentent et les conductivités diminuent. Le rôle des affluents est mis en évidence en comparant les profils hydrologiques de la Semoigne aux profils réalisés en soustrayant les apports latéraux issus du drainage de la couverture (fig. 4D-E). Pour les campagnes de septembre 2013 et de mars 2014, les affluents mesurés ne fournissent que $35 \%$ et $39 \%$ du débit observé à l'exutoire du bassin (Sem 9) pour chaque campagne de mesure, ce qui souligne un mode de vidange des aquifères préférentiellement concentré dans le corridor fluvial (tab. 1).

La carte des rendements spécifiques (fig. 5A-F), réalisée à partir des apports hydrologiques calculés pour chaque bassin élémentaire du réseau de mesure, confirme cette ob- 

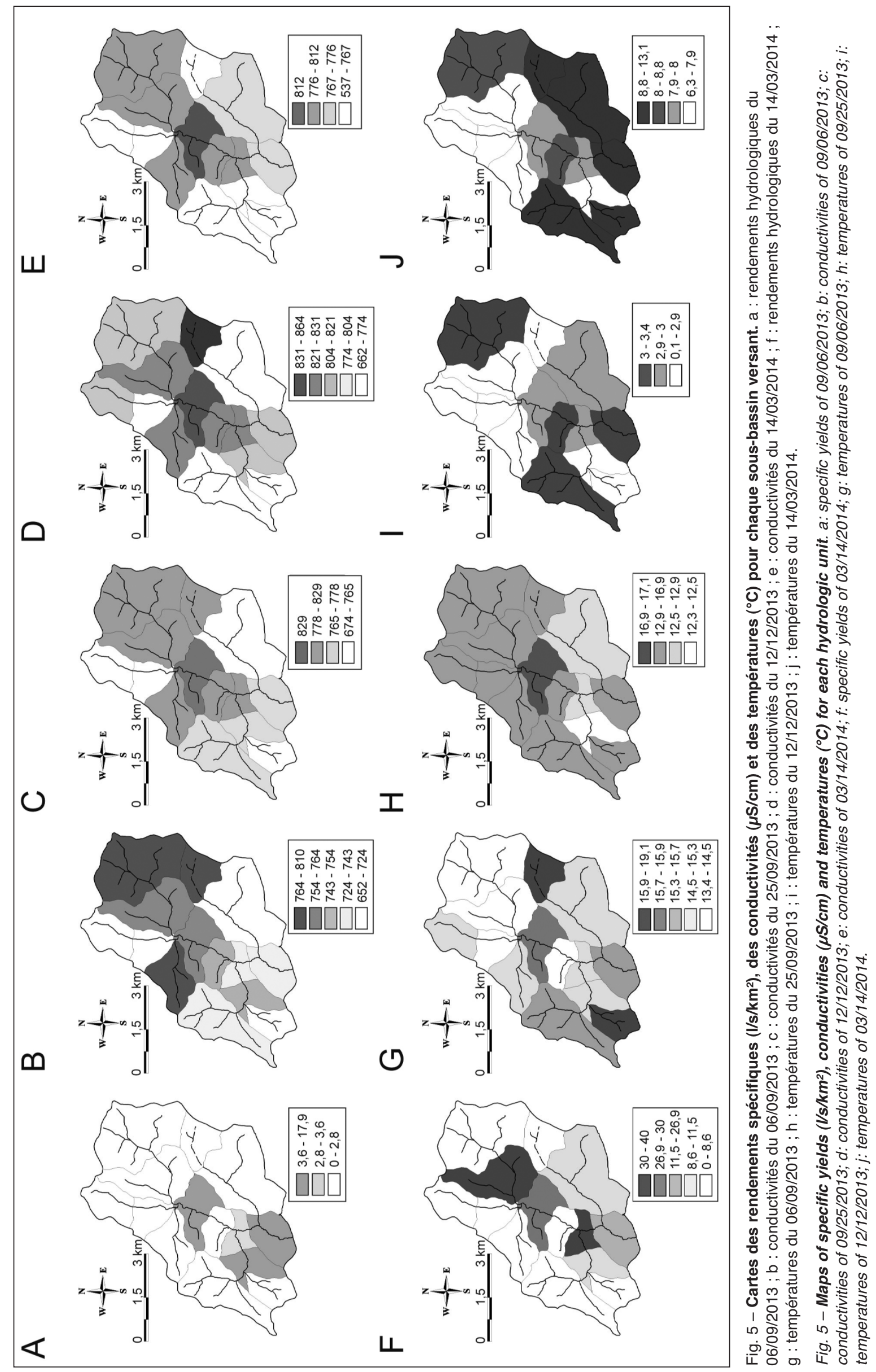


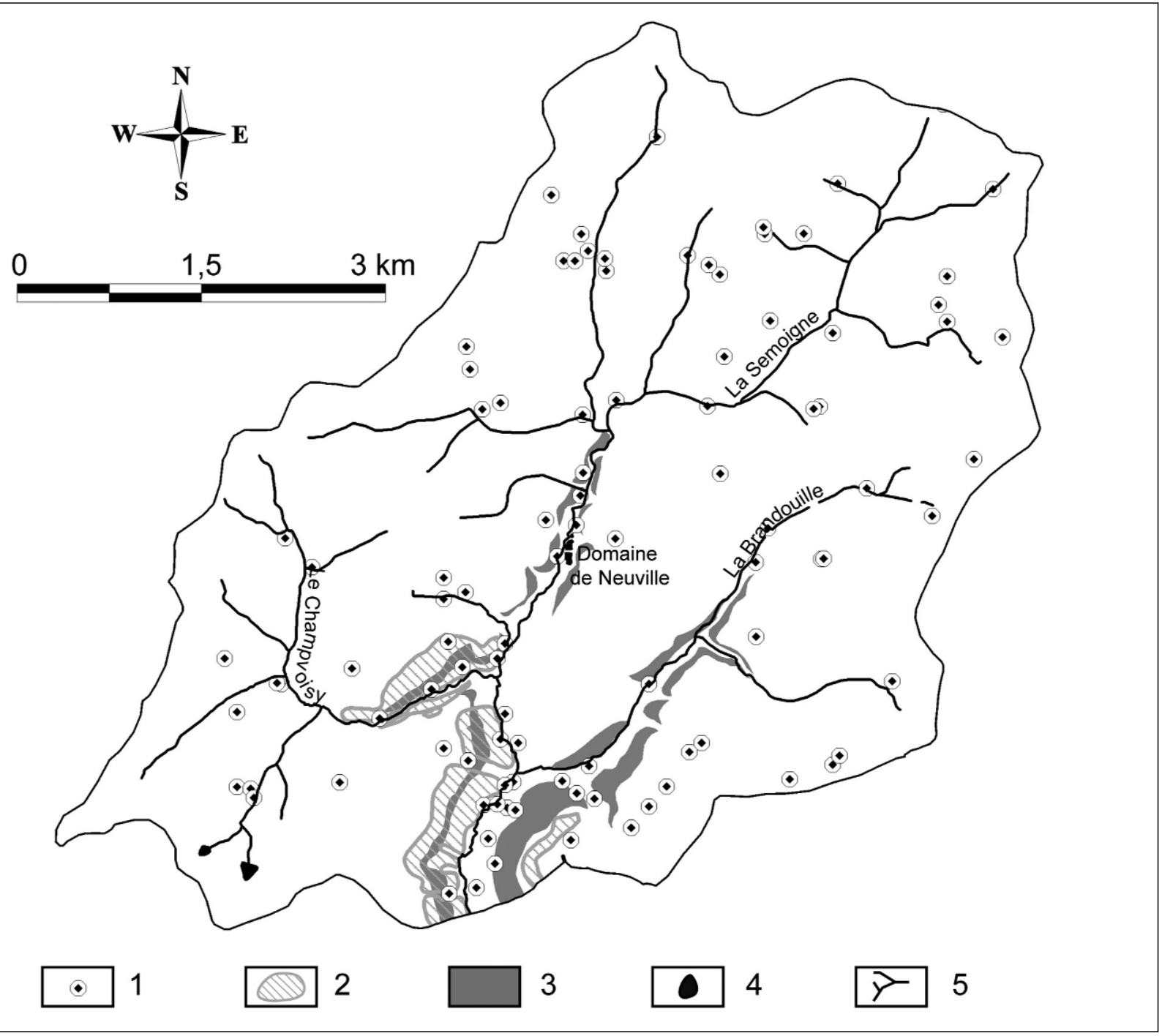

Fig. 6 - Carte des sources et emprise des glissements de terrain. 1 : source ; 2 : glissement de terrain ; 3 : aquifère principal ; 4 : étang ; 5 : cours d'eau.

Fig. 6 - Map of the springs and landslide areas. 1: spring; 2: landslide; 3: main aquifer; 4: pond; 5: stream.

\begin{tabular}{|l|c|c|c|c|}
\hline \multicolumn{1}{|c|}{ Campagne } & \multicolumn{2}{|c|}{$06 / 09 / 2013$} & \multicolumn{2}{c|}{$14 / 03 / 2014$} \\
\hline Affluents & $81 \mathrm{l} / \mathrm{s}$ & $35 \%$ & $328 \mathrm{l} / \mathrm{s}$ & $39 \%$ \\
\hline Corridor fluvial & $146 \mathrm{l} / \mathrm{s}$ & $65 \%$ & $511 \mathrm{l} / \mathrm{s}$ & $61 \%$ \\
\hline
\end{tabular}

Tab. 1 - Répartition des écoulements de la Semoigne à l'exutoire (Sem9).

Tab. 1 - Flow distribution of the Semoigne at the outlet (Sem9).

servation. La Semoigne supérieure (en amont de Sem 2), le Ruisseau de la Semoigne (Sem 3d), la Brandouille (Sem 8g), et le bassin supérieur du Ruisseau de Champvoisy (Sem 5d2 et Sem 5d3) présentent des rendements spécifiques faibles (1 à $\left.2,8 \mathrm{l} / \mathrm{s} / \mathrm{km}^{2}\right)$ qui contrastent avec ceux calculés en fond de vallée $\left(7\right.$ à 18 1/s/ $\left./ \mathrm{km}^{2}\right)$ entre les points Sem 4 et Sem 9.
C'est donc préférentiellement dans la vallée principale et dans la partie aval du bassin versant que la vidange aquifère s'effectue, là où les glissements sont les plus nombreux.

La cartographie des sources (fig. 6) montre un total de 97 sources dans l'ensemble du bassin versant, dont 71 (soit $73 \%$ ) sont des sources de dépression, c'est-à-dire localisées dans les vallées de la Semoigne et de ses affluents. Seulement 26 (soit $27 \%$ ) sont situées sur le plateau et, du fait de leur faible rendement, ne se raccordent pas, le plus souvent, au réseau hydrographique. Malgré une surface d'affleurement réduite (tab. 2), l'aquifère principal, très productif, compte 14 sources, ce qui représente une densité de plus de 5 sources par $\mathrm{km}^{2}$ d'affleurement, cinq fois supérieure à la densité moyenne pour l'ensemble du bassin versant de la Semoigne $\left(1,07\right.$ source $\left./ \mathrm{km}^{2}\right)$, où l'essentiel des sources émerge de la couverture $\left(0,94\right.$ source $\left./ \mathrm{km}^{2}\right)$. Ces valeurs montrent la prépondérance de l'aquifère de l'Yprésien supérieur/Lutétien inférieur et moyen dans les ressources en eau de la Semoigne, ainsi que son caractère capacitif et transmissif. 


\begin{tabular}{|c|c|c|c|}
\hline & Aquifère principal & $\begin{array}{c}\text { Couverture, aquicludes } \\
\text { et aquifères multicouches }\end{array}$ & $\begin{array}{c}\text { Bassin versant } \\
\text { de la Semoigne }\end{array}$ \\
\hline Superficie affleurement & $2,77 \mathrm{~km}^{2}$ & $88,23 \mathrm{~km}^{2}$ & $91 \mathrm{~km}^{2}$ \\
\hline Nombre de sources & 14 & 83 & 97 \\
\hline Densité de sources & 5,05 source $/ \mathrm{km}^{2}$ & 0,94 source $/ \mathrm{km}^{2}$ & 1,07 source $/ \mathrm{km}^{2}$ \\
\hline
\end{tabular}

Tab. 2 - Densité et nombre de sources selon les aquifères.

Tab. 2 - Density and number of springs according to aquifers.

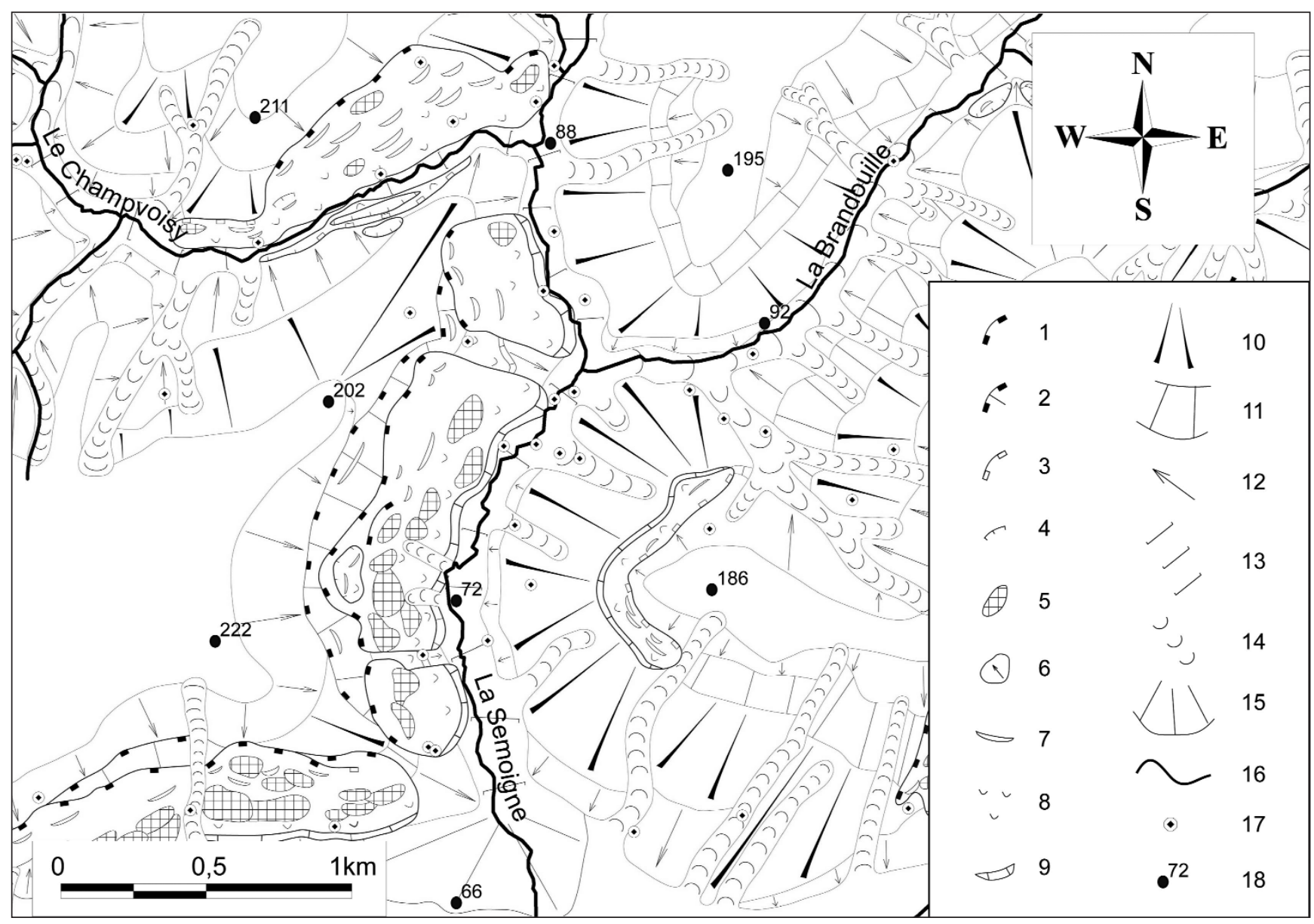

Fig. 7 - Croquis géomorphologique et répartition des sources. 1 : escarpement principal $; 2$ : escarpement régularisé ; $3:$ escarpement inférieur à $5 \mathrm{~m} ; 4$ : escarpement inférieur à $1 \mathrm{~m} ; 5:$ replat $; 6:$ contrepente $; 7:$ fluage $; 8:$ coulée $; 9:$ front de coulée $; 10:$ glacis $; 11$ : versant réglé ; 12 : versant de raccordement ; 13 : vallée à fond plat ; 14 : vallée en berceau ; $15:$ cône de déjection ; $16:$ cours d'eau ; 17 : source ; 18 : point côté.

Fig. 7 - Geomorphologic sketch and spring distibution. 1: main scarp; 2: graded scarp; 3: less than 5m scarp; 4: less than 1m scarp; 5 : bench; 6: reverse slope; 7: creep; 8: earth flow ; 9 : toe; 10: glacis; 11: graded hillslope; 12: hillslope; 13: flat valley floored; 14: cradle-shaped valley; 15: alluvial fan; 16: stream; 17: spring; 18: spot height.

La carte géomorphologique de l'aval du bassin versant (fig. 7) illustre l'ampleur des glissements de terrain dont les replats et/ou les contre-pentes atteignent des largeurs de $400 \mathrm{~m}$. Leur profondeur moyenne, une vingtaine de mètres, est déduite de leur morphologie et des données issues d'investigations (sondage, géophysique) faites sur d'autres glissements du secteur (Bollot, 2014). Les glissements se concen- trent en rive droite de la Semoigne sur le versant exposé à l'est, tandis que le versant exposé à l'ouest ne présente que des coulées superficielles affectant les formations du Lutétien supérieur et du Ludien. Ces coulées plus ou moins stabilisées, révélées par un modelé de fluage et des arbres dits en «queue de pipe », ne sont généralement pas mises en culture. Cette configuration est fréquente dans l'ensemble du 
vignoble champenois : l'organisation spatiale des glissements de terrain, dans cette structure homogène, s'explique par le sapement des rives concaves par les cours d'eau plutôt que par l'exposition conditionnant les alternances gel / dégel en période froide.

\section{Interprétation}

\section{Cartographie des ressources en eau}

La répartition des affleurements géologiques dans le bassin versant de la Semoigne (fig. 2 et fig. 6) conditionne les profils hydrologiques. Le segment supérieur de la Semoigne (amont de Sem 2) ainsi que ses affluents s'inscrivent dans les formations du Ludien (complexe supra-gypseux, Calcaire de Champigny, Calcaires et marnes de Saint-Ouen) et le Lutétien supérieur (Marnes et caillasses). Ces formations, lithologiquement hétérogènes et peu épaisses, constituent des aquifères multicouches en position d'interfluve et de plateau. Les faibles débits spécifiques mesurés illustrent la faible productivité de ces nappes perchées dont la surface piézométrique est fortement rabattue. La multiplicité des niveaux aquifères de ces formations détermine de nombreuses sources limnocrènes (associées à un plan d'eau) ou hélocrènes (marécageuses) sans connexion avec le réseau hydrographique. Ces sources peu productives entretiennent des zones humides de plateau caractéristiques de la Brie champenoise (fig. 8). Les formations ludiennes et les Marnes et caillasses s'apparentent donc à une compresse humide et jouent le rôle d'une couverture semi-perméable sur l'aquifère du Lutétien inférieur et moyen, alimenté par drainance. Ainsi, les fortes valeurs de conductivité relevées sur l'ensemble du bassin versant de la Semoigne s'expliquent par la solubilité du complexe supra-gypseux, qui libère des sulfates de calcium, et celle des Marnes et caillasses qui fournissent des eaux très minéralisées en carbonates de calcium à l'origine de nombreuses tufières de sources, des barrages de tufs, des ruisseaux tuffeux et des concrétions alluviales de type oncoïde (Devos et al., 2009). La saturation en $\mathrm{CaCO}_{3}$ des eaux qui pénètrent par drainance dans les calcaires du Lutétien moyen explique d'ailleurs le faible degré de karstification, généralement héritée et fossile, de ces calcaires (Devos et al., 2009, 2013). Pour comparaison, à Verneuil (équivalent du point Sem 9), le suivi de la qualité des eaux de la Semoigne dans le cadre du réseau national de bassin de l'Agence de l'Eau Seine-
Normandie montre une forte minéralité : de faciès bicarbonaté calcique, leur pH moyen est de 8,46 et leur dureté moyenne de $40^{\circ} \mathrm{F}$, avec des concentrations moins fortes en sulfates et en chlorures, $45 \mathrm{mg} / \mathrm{l}$ et $27 \mathrm{mg} / \mathrm{l}$ respectivement, pour la période $2010-2013$.

En revanche, dans sa partie médiane, la Semoigne recoupe les formations du Lutétien moyen (Calcaires grossiers et Tuffeau de Damery) et de l'Yprésien inférieur (Sables de Cuise). Ces formations plus homogènes et surtout plus perméables, parfois séparées par des niveaux argileux discontinus et peu épais (Argiles de Laon, $<1,5 \mathrm{~m}$ ), déterminent un aquifère unique de dépression qui constitue le principal réservoir en eau de la région. Il est beaucoup plus productif, ce qui se manifeste par l'augmentation marquée des débits spécifiques de Sem 4 à Sem 7 (fig. 4B et fig. 5A-F). La vidange de cet aquifère, captif sous les formations semi-perméables au niveau des plateaux, mais affleurant dans la vallée médiane de la Semoigne, s'effectue préférentiellement par le drain principal (cours d'eau drainant) et secondairement par des sources de fond de vallée en connexion directe avec le drain principal (sources d'émergence de nappe). La diminution modérée de la conductivité sur ce tronçon s'explique par une dilution (cet aquifère est plus productif), et par la présence des Sables de Cuise ; rappelons que les calcaires lutétiens, perchés et filtrants (leur nappe est généralement

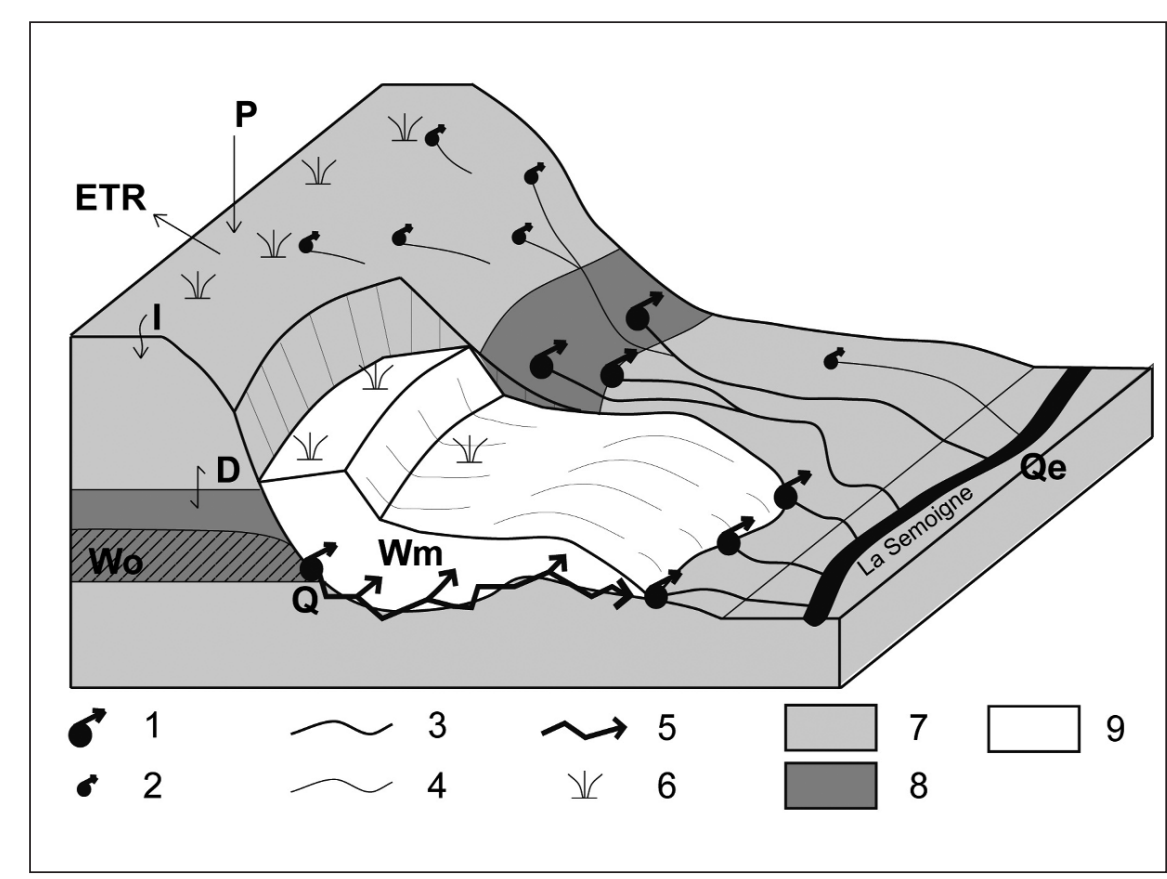

Fig. 8 - Schéma synthétique des ressources en eau. 1 : source de dépression de l'aquifère principal ; 2 : source de trop-plein perché ; 3 : cours d'eau drainant ; 4 : cours d'eau perdant ou endoréique ; 5 : circulation d'eau dans le glissement ; 6 : zone humide ; $7:$ formations semiperméables ; 8 : aquifère principal ; 9 : glissement de terrain ; $P$ : précipitations ; ETR : évapotranspiration réelle ; I : infiltration; $\mathrm{D}$ : drainance ; Wo : état de la réserve aquifère ; Wm : état hydrique des masses glissées ; $Q$ : débit aux sources ; Qe : débit d'étiage.

Fig. 8 - Synthetic diagram of water resources. 1: depression spring of main aquifer; 2: perched overflow spring; 3: draining river; 4: endorheic or water loss river; 5: water flow within the landslide; 6: wet area; 7: semi-permeable layers; 8: main aquifer; 9: landslide; P: rainfall; ETR: actual evapotranspiration; I: infiltration; D: leakage; Wo: aquifer water storage; Wm: landslide water storage; $Q$ : springs discharge; Qe: low flow. 
rabattue aux Argiles de Laon, là où ils existent), sont moins capacitifs que les sables cuisiens. Plus à l'aval, la Semoigne incise les formations argileuses de l'Yprésien inférieur qui constituent le mur de l'aquifère principal. Sur ce tronçon, la vidange aquifère ne s'effectue donc plus par des cours d'eau drainant mais par des sources latérales de fond de vallée ou de bas de versant (fig. 7). À la différence des sources du plateau, ces sources sont rhéocrènes, en connexion avec le drain principal, ce qui se manifeste par des débits spécifiques soutenus (entre Sem 7 et Sem 9).

Ces données hydrologiques permettent donc de spatialiser les ressources en eau et d'opposer les plateaux aux vallées. Dans cette structure quasi aclinale, l'organisation spatiale des ressources en eau dépend donc du degré d'incision des vallées. Les vallées peu incisées drainent une couverture semi-perméable peu productive au niveau des interfluves des plateaux. En revanche, les vallées profondes, proches de leur niveau de base local (la vallée de la Marne), déterminent une fenêtre géologique et recoupent l'aquifère captif et productif du Lutétien-Cuisien. Le long du corridor fluvial, la vidange aquifère s'effectue alors par drainage par les cours d'eau et par le biais d'émergences de dépression, au bas des versants et en fond de vallée. L'incision du réseau hydrographique a une autre conséquence sur l'organisation spatiale des ressources en eau : la dynamique de détente sur les versants, liée au creusement, se traduit par l'apparition d'un réseau de fissures parallèles à la vallée (Devos et $a l$. 2012) qui augmente la transmissivité du milieu.

\section{Ressources en eau et mouvements de terrain}

La cartographie géomorphologique montre que les glissements de terrain sont exclusivement positionnés dans la partie inférieure du bassin versant, marquée par les plus forts rendements hydrologiques de basses-eaux et d'étiage. Ils sont également localisés sur la principale ligne de sources, correspondant au contact entre l'aquifère du Lutétien-Cuisien et les argiles de l'Yprésien inférieur (fig. 6 et fig. 7), et leur présence, en retour, modifie la répartition des sources, qui se situent généralement à la périphérie des glissements : soit en bordure, là où le contact des sables du Cuisien et des argiles du Sparnacien affleure entre deux masses glissées ; soit au front des glissements, et donc en contrebas de la ligne de source potentielle. L'explication de cette dernière configuration par une continuité théorique entre aquifère du substrat et aquifère des glissements de terrain, qui ne rendrait d'ailleurs pas compte de l'absence de sources au sein des masses glissées, n'éclaire pas la question du comportement hydrogéologique des masses glissées. L'approche hydrologique apporte des éléments de réponse.

Dans le sous-bassin versant Sem 9 (fig. 3 et fig. 5), où le volume de l'aquifère Yprésien supérieur/Lutétien inférieur et moyen est seulement 5 fois supérieur à celui des glissements de terrain, l'influence de ces derniers sur les paramètres hydrologiques devrait être perceptible, ce qui n'est le cas ni pour la qualité des eaux ni pour les débits. Les glissements de terrain ne contiennent donc pas d'aquifère transmissif restituant régulièrement l'eau via une source rhéocrène. Si leur rôle de réservoir est possible, l'hétérogénéité du matériel déplacé et déstructuré contrarie la circulation de l'eau. L'écoulement pérenne des sources en contrebas des glissements n'en est donc que partiellement issu, et l'alimentation de ces sources est donc liée à la vidange régulière de l'aquifère principal le long du plan de glissement (fig. 8). La masse glissée joue donc un rôle de couverture semi-perméable (voire imperméable), barrant un aquifère principal devenu semi-captif. Une infiltration remontante au sein du glissement est cependant possible comme l'indiquent les données piézométriques (Bollot, 2014), conditionnant davantage une pression interstitielle à l'origine de la réactivation que la formation d'une nappe sensu stricto. À l'infiltration remontante s'ajoute probablement l'eau infiltrée des précipitations (dans une proportion non calculée). Les masses glissées provoquent donc sur les terrains qu'elles recouvrent une véritable discontinuité dans le champ des perméabilités. Ainsi, en rive droite de la Semoigne inférieure, les affleurements aquifères sont-ils drapés d'une couverture peu perméable à l'origine d'anomalies dans la position des sources, tandis qu'en rive gauche, en l'absence de mouvements de terrains, les eaux sourdent le long des lignes de sources potentielles ou selon le dispositif hydrogéologique d'aquifère à surface libre.

\section{Discussion}

Les relations entre ressources en eau et mouvements de terrain témoignent donc du rôle important des eaux souterraines dans l'organisation spatiale des glissements. Cette adéquation peut donc nous renseigner sur les conditions d'écoulement durant la genèse des glissements et, partant, sur les conditions environnementales dans lesquelles ils se sont produits. L'état des ressources en eau souterraine dépend du bilan hydrologique et plus particulièrement des phases de surplus hydrologique garantissant une bonne infiltration. À cette latitude, les conditions d'infiltration optimales - évapotranspiration réelle modérée, pluies efficaces élevées - se réalisent en phase cataglaciaire, favorisées par la fonte du pergélisol, puis s'estompent sous climat tempéré, où le déficit hydrique (et hydrologique) s'accentue. La déstabilisation des versants est donc synchrone de la dégradation du pergélisol et de la reconstitution de l'aquifère, que T. Pánek et al. (2010), en Slovaquie, datent du passage du Tardiglaciaire à l'Holocène. Les mouvements de terrain « anciens » du vignoble champenois, qui provoquent des anomalies dans la position des sources, seraient donc d'âge pléistocène, sans être pour autant préglaciaires (Van den Eeckhaut et al., 2010) puisque le long de la Semoigne les masses glissées atteignent le fond de la vallée, qui lui-même se raccorde à la plaine alluviale de la Marne, donc se rattache au dernier épisode de creusement. Les quelques vallons secs qui incisent les masses glissées indiquent quant à eux que les mouvements de terrain seraient précoces sur les versants, où la dégradation du pergélisol est plus rapide (Van Vliet-Lanoë, 1988). Cette succession mouvement de masse- 
ruissellement de surface est d'ailleurs typique d'une séquence morphogénique parapériglaciaire (Mercier, 2008).

Ce constat incite à ajouter, dans les modèles de susceptibilité des versants aux mouvements de terrain, le paramètre « eaux souterraines » en tant que facteur d'instabilité (Schmidt et Dikau, 2004 ; Schulz et al., 2008), sans se limiter à l'état hydrique des masses glissées, et en tenant compte de l'alimentation souterraine en eau. Dans le vignoble, cette couche d'information n'a jamais été analysée, sans doute en raison de la rareté des banques de données numériques disponibles. De fait, les paramètres « lithologie » et « pente » apparaissent toujours, in fine, comme les plus discriminants sur les cartes de susceptibilité (Bălteanu et al., 2010 ; Van den Eeckhaut et al., 2010 ; Conforti et al., 2014). Pour ce qui est de la pente, il est pourtant notoire que les mouvements de terrain ne sont pas l'apanage des reliefs de forte énergie (Van den Eeckhaut et al., 2007) et, de plus, la pertinence de ce paramètre est discutable quand les cartes de susceptibilité portent sur des secteurs où les mouvements de terrains ont déjà eu lieu et définissent une valeur de pente qui, en toute logique, traduit un équilibre retrouvé. Ce sont donc les modalités des réactivations qu'il faut établir.

À ce titre, le croisement des valeurs standardisées des niveaux piézométriques mesurés dans les calcaires du Lutétien inférieur et moyen à Armentières-sur-Ourq et dans les sables de l'Yprésien supérieur à Acy (Banque de Données ADES), de l'écart de la somme cumulée à la moyenne (CuSum) des précipitations mensuelles à Epernay (données du Comité Interprofessionnel du Vin de Champagne), et de l'occurrence des mouvements de terrain dans le secteur du vignoble champenois (Banque de Données MVT du BRGM), montre que les totaux pluviométriques importants n'induisent pas systématiquement des mouvements de terrain (fig. 9). L'aléa est quasi inexistant lors d'années exceptionnellement pluvieuses mais

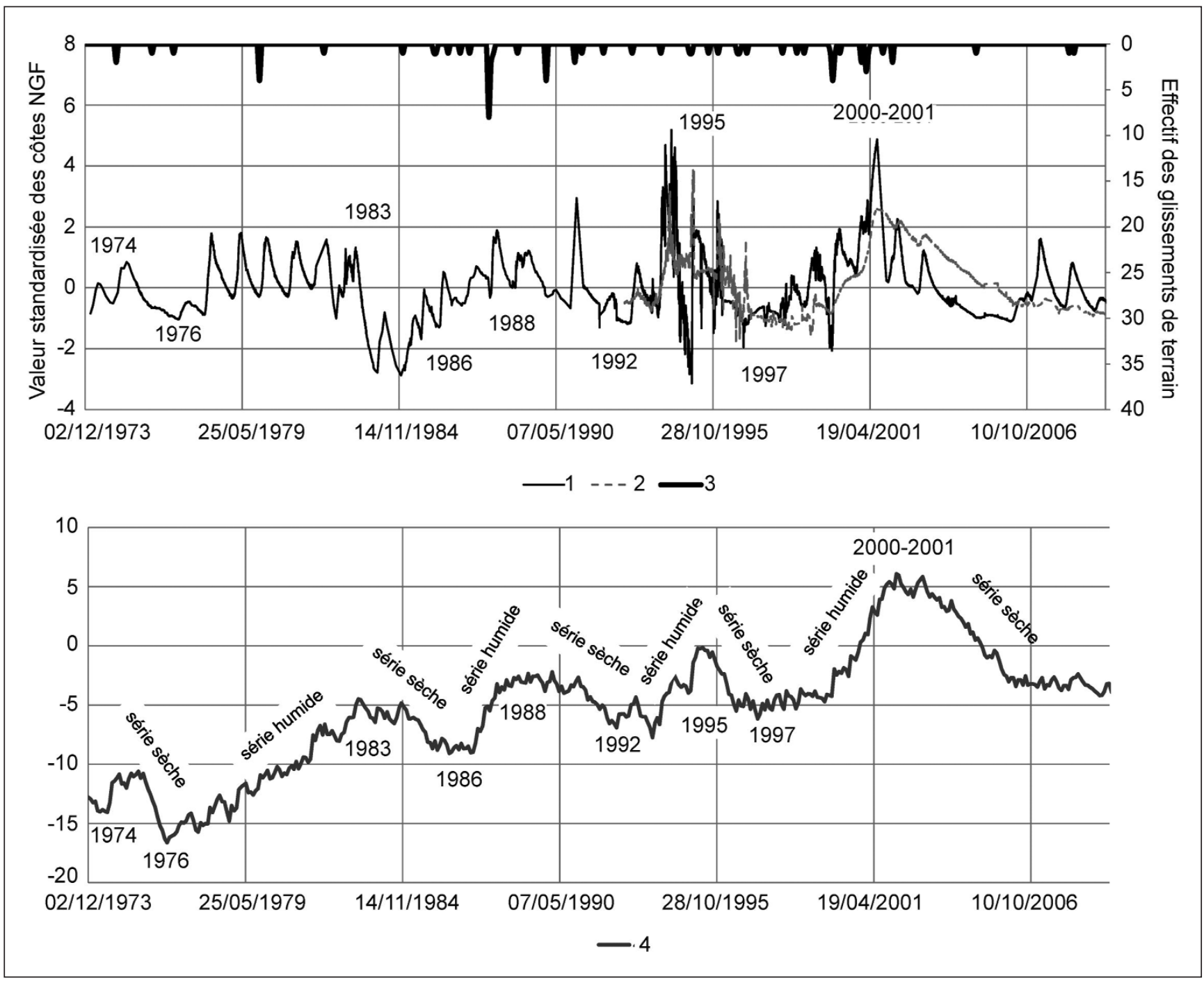

Fig. 9 - CuSum des précipitations, niveaux de nappes et nombre de glissements de terrain. 1 : piézomètre d'Armentières sur Ourcq (Lutétien) ; 2 : piézomètre d'Acy (Yprésien supérieur) ; 3 : effectif annuel de mouvements de terrain ; $4:$ CuSum des précipitations à Epernay.

Fig. 9 - CuSum of the rainfalls, groundwater levels and number of landslides. 1: piezometer of Armentières sur Ourcq (Lutetian ; 2: piezometer of Acy (upper Ypresian); 3: annual number of landslides; 4: CuSum of the rainfall in Epernay. 
isolées au sein de séries d'années sèches (1974, 1992). De même, la succession d'une série d'années sèches (19711976), et d'une série d'années plus humides (1977-1983) est peu morphogène. Dans ce cas, partant d'un niveau très bas, la recharge détermine des rendements aquifères insuffisants, peu propices à l'alimentation en eau des masses glissées et au déclenchement de nouveaux glissements. Cela témoigne en outre du faible rôle de la dessiccation de surface en année sèche, qui augmente pourtant la perméabilité des matériaux (souvent de nature argileuse) et l'infiltration en année humide. En revanche, les mouvements de terrain sont plus nombreux au terme d'une succession d'années humides garantissant une recharge aquifère prolongée, comme de 1986 à 1988 et en 2000-2001 (où ont été enregistrés des niveaux piézométriques records et où se sont produit des crues de nappe). L'instabilité s'accentue également si une série d'années humides se greffe sur une période où les niveaux piézométriques sont restés élevés, comme ce fut le cas entre 1995 et 1997. Logiquement, une série d'années sèches engendre très peu, voire aucun mouvement de terrain.

En conséquence, l'état hydrique des masses glissées (Wm) ne découle pas exclusivement du bilan climatique (P - ETR) mais davantage de l'état des réserves aquifères (Wo) qui leurs confèrent une alimentation souterraine non seulement continue (Q) mais également immunisée de l'évapotranspiration (fig. 8). Dans le vignoble, pourtant, les désordres actuels qui affectent les versants, essentiellement des réactivations superficielles d'anciens glissements (le glissement de Rilly-la-Montagne constituant l'exception ; Marre, 1987), sont généralement attribuées aux précipitations et à un bilan hydrique chroniquement défavorable à la stabilité des versants (Guéremy et Vejux, 1987). Il n'en reste pas moins que les fluctuations climatiques à l'échelle de l'Holocène ont rythmé la dynamique des versants, avec une recrudescence marquée de l'instabilité à la fin de l'Atlantique (González-Díez et al., 1999 ; Demoulin et al., 2003 ; Soldati et al., 2004 ; Pánek et al., 2010), période à partir de laquelle paramètres anthropiques et climatiques commencent en outre à se combiner (Dapples et al., 2002 ; Remondo et al., 2005 ; Van den Eeckhaut et al., 2010). L'exemple de la Semoigne incite cependant à ne pas raisonner uniquement en termes de bilan hydrique : dans le vignoble - où les aménagements sont voués à faciliter (et intensifier) l'exploitation plus qu'à stabiliser les pentes les réactivations sont dues à la teneur en eau des masses glissées, alimentées davantage par la vidange aquifère que par les précipitations, qui ne peuvent donc être utilisées comme le seul critère de déclenchement des mouvements de terrain.

\section{Conclusion}

L'approche hydrologique des eaux de surface en période de basses eaux et d'étiage permet de spatialiser les ressources en eau au sein des bassins versant et de comprendre le rôle fondamental des eaux souterraines dans l'organisa- tion spatiale des mouvements de terrain. Il faut donc distinguer le comportement de l'aquifère d'une part, relevant du bilan hydrologique et influençant la stabilité de versants, et le rôle indirect des précipitations, relevant du bilan hydrique, dont l'efficacité est bien moindre. En ne prenant pas en compte l'aquifère, la cartographie de la susceptibilité aux glissements pour le vignoble champenois (Van den Eeckhaut et al., 2010) surestime finalement, dans le temps mais aussi dans l'espace, la probabilité d'un mouvement de terrain. Si la pente et la lithologie semblent conserver une certaine pertinence, c'est que la Semoigne, pour rattraper son niveau de base, a plus incisé dans sa partie aval, où se concentrent les glissements affectant l'Yprésien et le Lutétien inférieur et moyen. Mais, en termes de stabilité, la conséquence de cette incision est le recoupement de l'aquifère principal, dont la vidange est la cause première des volumineux mouvements de masse de phase cataglaciaire, et de leur réactivation superficielle ultérieure. Bien que dans les conditions bioclimatiques actuelles les eaux souterraines alimentent moins les masses glissées, la modélisation spatiale des écoulements d'étiage (Lang et al., 2006) pourrait être intégrée aux modèles prédictifs de réactivation de mouvements de terrain. Par ailleurs, le suivi hydrométrique et géochimique des sources en limite de coulées permettrait de mieux connaître les contraintes de cheminement des eaux et les conditions d'écoulement souterrains entre les aquifères et les masses glissées.

\section{Références}

Bălteanu D., Chendeş V., Sima M., Enciu P. (2010) - A countrywide spatial assessment of landslide susceptibility in Romania. Geomorphology 124, 102-112.

Belle P., Aunay B., Bernardie S., Grandjean G., Ladouche B., Mazué R., Join J.-L. (2013) - The application of an innovative inverse model for understanding and predicting landslide movements (Salazie cirque landslides, Reunion Island). Landslides, DOI 10.1007/s10346-013-0393-5.

Bollot N. (2014) - Les mouvements de terrain du vignoble champenois. Approche géomorphologique et hydrologique. Thèse de doctorat, Université de Reims Champagne-Ardenne, 2 tomes, $343 \mathrm{p}$.

Bollot N., Devos A., Pierre G., Chalumeau L., Lejeune, O. (2014) - Détente mécanique et dynamique de versant dans l'Eocène de l'est du bassin de Paris, France. Géomorphologie : relief, processus, environnement, 2, 121-132.

Compagnon F., Guglielmi Y., Mudry J., Follaci J.-P., Ivaldi J.P. (1997) - Approche chimique et isotopique de l'origine des eaux en transit dans un grand mouvement de terrain : exemple du glissement de la Clapière (Alpes-Maritimes, France). C. $R$. Académie des Sciences de Paris, 325, 565-570.

Conforti M., Pascale S., Robustelli G., Sdao F. (2014) - Evaluation of prediction capability of the artificial neural networks for mapping landslide susceptibility in the Turbolo River catchment (northern Calabria, Italy). Catena 113, 236-250.

Dapples F., Lotter A.F., van Leeuwen J.F.N., van der Knaap W.O., Dimitriadis S., Oswald D. (2002) - Paleolimnological 
evidence for increased landslide activity due to forest clearing and land-use since $3600 \mathrm{cal} \mathrm{BP}$ in the western Swiss Alps. Journal of Paleolimnology 27, 239-248.

Demoulin A,.Pissart A., Schroeder C. (2003) - On the origin of late Quaternary palaeolandslides in the Liège (E Belgium) area. International Journal of Earth Sciences 92, 795-805.

Devos A., Jaillet S., Gamez P. (1999) - Structures tectoniques et contraintes de cheminement des eaux dans les aquifères karstiques du Barrois. Geodinamica Acta 12, 3-4, 249-257.

Devos A., Lejeune O., Chopin E. (2007) - Structural control on surface flow in karstic environnement. Geodinamica Acta 20, 393-402.

Devos A., Sosson C., Fronteau G., Lejeune O. (2009) - Les tufières du Vormy et des Fontinettes (Aisne-Marne-France) : marqueurs de la faible karstification des calcaires lutétiens de l'Est du Bassin parisien ? Karstologia, 54, 37-48.

Devos A., Chalumeau L., Bollot N., Sosson C., Barre E., Lejeune O., Gobance G. (2012) - Organisation spatiale de la fracturation de détente mécanique en carrières souterraines -application aux plateaux éocènes du bassin de Paris. PhysioGéo, 6, 243-262.

Devos A., Sosson C., Lejeune O., Chalumeau L., Chopin E. (2013) - The role of geomorphological control of aquifer on exchanges between water table and river flow in limestone plateau from eastern Paris basin. In Actes des journées hydrologiques, Commission des hydrosystèmes continentaux, IUKB, Sion, Suisse, Ed. Pfeil (Munich, Allemagne), 313-322.

Dewolf Y., Pomerol C. (1997) - Surfaces d'érosion polygéniques dans les formations cénozoïques du Bassin parisien. Bulletin de la Société Géologique de France, 4, 473-482.

González-Díez A., Remondo J., Díaz de Terán J.R., Cendrero A. (1999) - A methodological approach for the analysis of the temporal occurrence and triggering factors of landslides. Geomorphology 30, 95-113.

Grandjean G., Pennetier C., Bitri A., Meric O., Malet J.-P. (2006) - Caractérisation de la structure interne et de l'état hydrique de glissements argilo-marneux par tomographie géophysique : l'exemple du glissement-coulée de Super-Sauze (Alpes du sud, France). C. R. Geoscience, 338, 587-595.

Grandjean G., Hibert C., Mathieu F., Garel E., Malet J.-P. (2009) - Monitoring water flow in a clay-shale hillslope from geophysical data fusion based on a fuzzy logic approach. C.R. Geoscience 341, 937-948.

Guéremy P., Vejux V. (1987) - Mouvements de terrain successifs : les glissements de terrain et les coulées du versant sud de la Montagne à Avize (Marne, France). Travaux de l'Institut de Géographie de Reims, 69-72, 113-127.

Guglielmi Y., Cappa F., Binet S. (2005) - Coupling between hydrogeology and deformation of mountainous rock slopes: Insights from La Clapière area (southern Alps, France). C. R. Geoscience 337, 1154-1163.

Lang C., Gille E., François D., Auer J.C. (2006) - Le dispositif PRESAGES : un ensemble d'outils pour la prévision des étiages. In IAHS publication 308, Climate Variability and Change Hydrological Impacts, 145-150.

Lebourg T., Hernandez M., Zerathe S., El Bedoui S., Jomard H., Fresia B. (2010) - Landslides triggered factors analysed by time lapse electrical survey and multidimensional statistical approach. Engineering Geology 114, 238-250.

Lee C.C., Yang C.H., Liu H.C., Wen K.L., Wang Z.B., Chen Y.J. (2008) - A study of the hydrogeological environement of the lishan landslide area using resistivity image profiling and borehole data. Engineering Geology 98, 115-125.

Lejeune O., Devos A. (2004) - Apport des méthodes hydrologiques dans la compréhension des écoulements en Pays calcaires : exemple des bas plateaux jurassiques du haut bassin de la Marne (France). Karstologia, 44, 15-24.

Lissak C., Maquaire O., Malet J.-P. (2009) - Role of hydrological process in landslide occurrence : Villerville-Cricqueboeuf landslides Normandy coast, France. In Malet J.-P., Remaître A., Boogard T.A. (Ed.): Proceedings of the International Conference on Landslide Processes: from geomorphologic mapping to dynamic modelling, Strasbourg, CERG Éditions, 175180.

Marre A. (1987) - Le mouvement de terrain du 23 août 86 à Rillyla-Montagne (51). Naissance et évolution. Travaux de l'Institut de Géographie de Reims, 69-72, 95-111.

Mercier D. (2008) - Paraglacial and paraperiglacial landsystems: concepts, temporal scales and spatial distribution. Géomorphologie, relief, processus, environnement, 4, 223-234.

Pánek T., Hradecký J., Smolková V., Šilhán K., Minár J., Zernitskaya V. (2010) - The largest prehistoric landslide in northwestern Slovakia: Chronological constraints of the Kykula long-runout landslide and related dammed lakes. Geomorphology 120, 233-247.

Parriaux A. (2010) - Hydrogéologie et glissements de terrain. Gas Wasser und Abwasser, 11, 978-985.

Pierre G., Lahousse P. (2006) - The role of groundwater in cliff instability: an example at Cape Blanc-Nez (Pas-de-Calais, France). Earth Surface Processes and Landforms 31, 31-45.

Remondo J., Soto J., Gonzalez Diez A., Diaz de Teran J.R., Cendrero A. (2005) - Human impact on geomorphic processes and hazards in mountain areas in northern Spain. Geomorphology 66, 69-84.

Ruiz-Villanueva V., Bodoque J.M., Díez-Herrero A., Calvo C. (2011) - Triggering threshold precipitation and soil hydrological characteristics of shallow landslides in granitic landscapes Geomorphology 133, 178-189.

Schmidt J., Dikau R. (2004) - Modeling historical climate variability and slope stability. Geomorphology 60, 433-447.

Schulz W.H., Lidke D.J., Godt J.W. (2008) - Modeling the spatial distribution of landslide-prone colluvium and shallow groundwater on hillslopes of Seattle, WA. Earth Surface Processes and Landforms 33, 123-141.

Soldati M., Corsini A., Pasuto A. (2004) - Landslides and climate change in the Italian Dolomites since the Late glacial. Catena 55, 141-161.

Umar Z., Pradhan B., Ahmad A., Jebur M.N., Tehrany M.S. (2014) - Earthquake induced landslide susceptibility mapping using an integrated ensemble frequency ratio and logistic regression models in West Sumatera Province, Indonesia. Catena 118, 124-135.

Van Den Eeckaut M., Verstraeten G., Poesen J. (2007) - Morphology and internal structure of a dormant landslide in a hilly 
area: The Collinabos landslide (Belgium). Geomorphology 89, 258-273.

Van Den Eeckaut M., Marre A., Poesen J. (2010) - Comparison of two landslide susceptibility assessments in the ChampagneArdenne region (France). Geomorphology 115, 141-155.
Van Vliet-Lanoë B. (1988) - Le rôle de la glace de ségrégation dans les formations superficielles de l'Europe de l'Ouest. Processus et héritages. Editec, Caen, 854 p.

Article soumis le 18 avril 2014, accepté le 20 octobre 2014. 